\title{
An Ultra Small Angle Scattering Study of Hydrogen in Metals
}

\author{
D. Bader, H. Rauch \\ Atominstitut der Österreichischen Universitäten, A-1020 Wien \\ and A. Zeilinger \\ Department of Physics, Massachusetts Institute of Technology, Cambridge, MA 02139, U.S.A.
}

Z. Naturforsch. 37a, 512-516 (1982); received March 29, 1982

Dedicated to Prof. G. Hildebrandt on the occasion of his 60th birthday

A diffractometer using perfect crystals in symmetric Bragg arrangement, hence being useful for both neutron and X-ray radiation, has been built. The angular resolution of that instrument is better than 1 microradian, which corresponds to a scattering vector resolution of about $10^{-6} \AA^{-1}$. This spectrometer was equipped with a laser light adjustment system which is sensitive to the relative angular position of the two perfect crystals. The spectrometer was used for the measurement of neutron scattering at very low angles of hydrated niobium and vanadium samples. The experimental results show an anomalously low attenuation of the neutrons when the metal- $\mathrm{H}$ sample is within the solubility $\alpha$ region. This property is explained by a lattice gas model of atomic $\mathrm{H}$ in the $\alpha$-phase of the system. In the two phase region $(a-\beta)$ a stronger attenuation of the neutron beam occurs due to the sample now being in an ordered interstitial solution phase.

\section{Introduction}

Small angle scattering spectrometers being a standard tool in neutron physics have in recent years been extended to the high angle resolution regime by employing perfect crystals in a nondispersive double crystal arrangement $[1-4]$. The nondispersive property of a parallel setting of the Braggplanes of the two crystals permits to operate such spectrometers with a good neutron economy. The wavelength spread as determined by the collimation geometry does not to first order influence the Q-resolution. In such an arrangement the sample to be investigated is placed between the two Bragg-diffracting crystals and the change in angular distribution of the radiation after passage through the sample is recorded by scanning the second crystal. Due to the high angle resolution of a perfect crystal small angle scattering device, for many experiments it is possible to reach the refraction domain as approach to the diffraction domain with respect to the sample, i. e. one measures primarily the modification of the angular distribution of the direct beam and the low-angle transmission effect defined as

$$
I=I_{0} \exp \left(-N_{\mathrm{p}} \sigma_{\mathrm{sas}} t\right)
$$

Work supported by Fonds zur Förderung der wissenschaftlichen Forschung, Project 3836.

Reprint requests to Dr. D. Bader, Atominstitut der Österreichischen Universitäten, A-1020 Wien. where $N_{\mathrm{p}}$ is the number of precipitates per volume and $\sigma_{\mathrm{sas}}=\pi R^{4}\left(N_{\beta} b_{\beta}-N_{\alpha} b_{\alpha}\right)^{2} \lambda^{2} / 2$ is the scattering cross section of a precipitate of radius $R$ and scattering density $N_{\beta} b_{\beta}$ in a matrix with a scattering density $N_{a} b_{a}$ [5]. A previous investigation in this direction has been done by Kalanov et al. [6] using a similar technique.

The present paper describes the design characteristics of such an instrument as setup at the TRIGA reactor Wien. The instrument was used for studying metal samples loaded with hydrogen and deuterium at room temperature in order to study precipitation effects. Another experiment carried out with that instrument concerned Christiansen filter measurements with the aim of providing a complementary technique to measure neutron scattering lengths [7].

\section{The Instrument}

For an infinitely thick non absorbing perfect crystal the reflectivity is given by the well-known Darwin function $[8,9]$

$$
R(y)=\begin{aligned}
& 1 \\
& 1-\sqrt{1-y^{-2}},
\end{aligned},|y| \geqq 1,
$$

where in the symmetric Bragg-case

$$
y=\frac{\left[\left(\theta-\theta_{\mathrm{B}}\right) \sin 2 \theta_{\mathrm{B}}-V(0) / E\right]}{[V V(\mathbf{G}) V(-\mathbf{G}) / E]} .
$$


Here, $\theta$ is the angle of the direction of the incident neutron beam, $\theta_{\mathrm{B}}$ is the exact Bragg angle, $V(0)$ and $V(\boldsymbol{G})$ are the mean and the Fourier components of the neutron-crystal interaction potential and $E$ is the neutron kinetic energy. In this experiment the (400) reflection at Si $\left(V(400) \cong V(0)=2 \pi \hbar^{2} b_{\mathrm{c}} N / m\right.$, $b_{\text {c }}$ coherent scattering length, $N$ particle density) at a Bragg-angle of $41.21^{\circ}$ is used and we expect the total reflection range to be $\Delta \theta=4.0 \times 10^{-6}$ radians.

An additional reduction of the angular width of the rocking curve can be achieved by cutting the crystals with a proper angle relative to the reflecting planes [1]. A possibility to reduce the tails of the resolution function exists in the use of multiple reflection by groove crystals as successfully applied to $\mathrm{X}$-ray small angle cameras $[10,11]$.

The small reflection width implies the necessity of a very high angular stability and a fine angular control of the experiment. The stability requirement was met by having both crystals mounted on an optical bench which was thermally isolated. In order to obtain the necessary angle resolution, a commercially available theodolite (WILD T2) was used as a goniometer similar as done previously for an $\mathrm{X}$-ray setup [12]. The angular position itself was controlled by driving the fine angle setting knobs of the theodolite by a stepping motor in such a way that one step of the stepping motor provided a rotation of the crystal by $1 / 30$ arcsec. It is noteworthy that the whole angular range accessible by that high resolution stepping mode was $5^{\circ}$. This setup was used for the analyzing crystal, while the monochromator crystal, which was bathed in the white neutron beam emerging from a reactor beam tube, was mounted on a standard goniometer head.

In order to obtain a measurement of the angular position of the crystals, a laser mirror system was developed (Figure 1 [7]). This system employs two mirrors each connected to one of the crystals. A laser beam is reflected consecutively by both these mirrors onto a reference mirror which reflects it back into the same ray path in such a way that finally the position sensitive diode located at the laser given a measurement of the angular deflection of the laser beam. It can be shown that, if the reference mirror is arranged orthogonal to the beam leaving the laser source, the total angular deflection when the beam returns to the position sensitive diode is $4 \varphi$, where $\varphi$ is the misstepping of the second mirror with respect to the first one. To overcome the
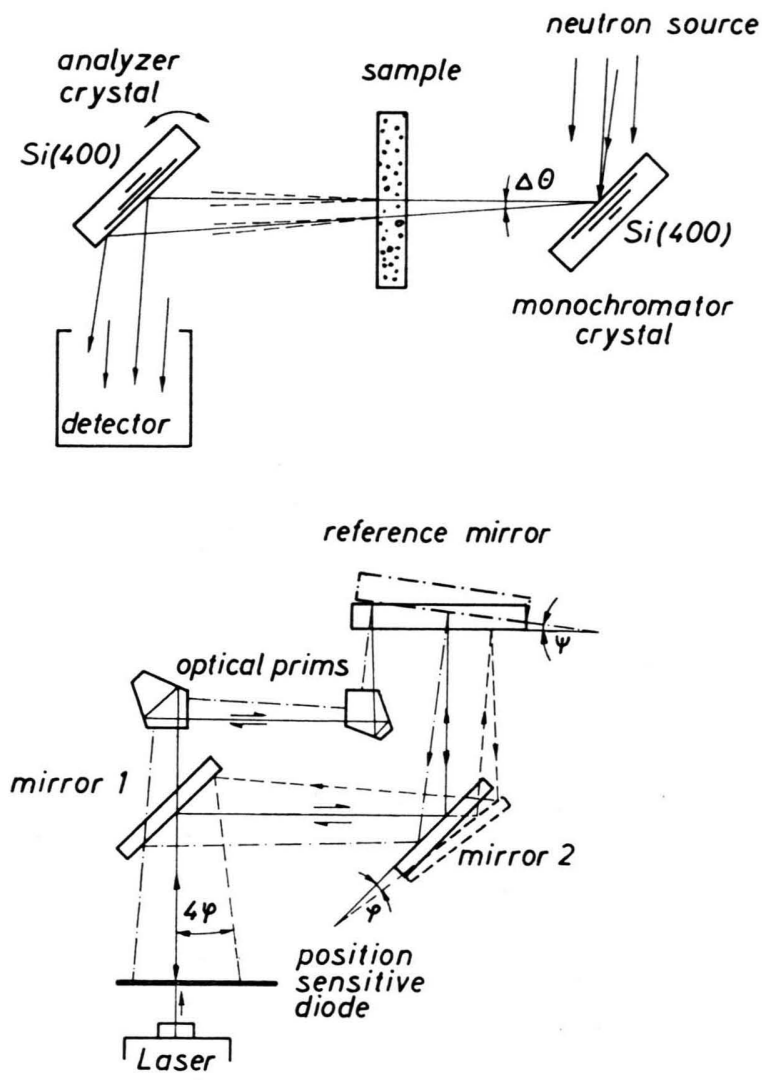

Fig. 1. Sketch of the nondispersive double crystal spectrometer for small angle scattering studies (top) and the optical adjustment system (below).

sensitivity of the system to the angular position of the reference mirror a reference beam was guided along a parallel beam path using pentagonal prisms which deflect by the fixed angle of $90^{\circ}$. Then the positional separation $\delta x$ between the illuminated spot of the measuring beam and of the reference beam is given as $\delta x=4 \varphi l$, where $l$ is the optical path length. Using a focussing mirror as the reference mirror, an angular resolution down to $\sim 10^{-7}$ radians was achieved. As compared to a light interferometeer arrangement [13], our system has - besides its simplicity - the advantage of giving an absolute reading of the relative angle, which is not lost by switching off the system. Figure 2 shows the measured double crystal rocking curve for neutrons. The experimental full width at half maximum of $11 \mu \mathrm{rad}$ was found to be only slightly larger than the theoretically expected full width $(8 \mu \mathrm{rad})$. This experimental result was obtained after optimization of 


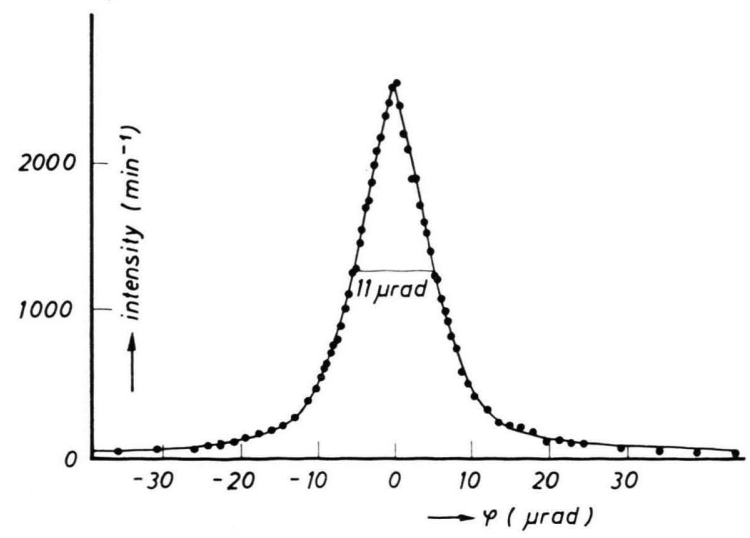

Fig. 2. Rocking curve (= resolution function) of the perfect crystal arrangement.

the $\varrho$-axis positioning of the crystals, because any missetting $\Delta \varrho$, together with a vertical divergency $\Delta \alpha$, gives a broadening of $\Delta \varrho^{2} /\left(2 \cos ^{2} \theta_{\mathrm{B}}\right)+$ $\Delta \varrho \Delta \alpha \sin \theta_{\mathrm{B}}$.

\section{Experiment and Results}

The experiments were done by using sets of samples of $V$ loaded with $\mathrm{H}$ and $\mathrm{D}$, respectively, and of $\mathrm{Nb}$ loaded with $\mathrm{H}$. These samples covered a range of concentrations from very low concentrations to values beyond the $\alpha$-phase boundary. Some of these samples had been investigated and specified in an earlier experiment by neutron interferometry [14].

Figure 3 shows the characteristic results for the measurements of the hydrogen loaded niobium samples. Note that below the $\alpha$-phase boundary the rocking curves do not exhibit a significant change of their shape when the hydrogen concentration is increased. This behavior changes drastically above the phase boundary. Here the width of the rocking curves increases with increasing $\mathrm{H}$ concentration due to the expected precipitates of the $\beta$-phase. It was not attempted in the present work to derive from the broadening effect information about the size of the precipitates, because this needs a rather complicated deconvolution procedure including the considerable vertical divergency $[15,16]$ and the tails of the resolution function (Figure 2). On a relative scale it can be seen that a linear Guinier plot cannot be obtained indicating rather different size of the precipitates.

An interesting result is obtained if one plots the integrated intensity of the small angle scattering peak as a function of hydrogen concentration (Figure 4). Here the integrated intensity stays again fairly constant below the phase boundary and decreases markedly beyond that boundary. In order to obtain the integrateed intensities, a computer convolution procedure was employed. The resolution function as shown in Fig. 2 has been approximated by a convolution function of two Darwin reflection curves with a variable width parameter. This function was again convoluted with the scattering function, assuming that it is of Lorenzian or Gaussian shape. A proper fit procedure yielded the parameters of the assumed scattering law. A quantitative interpretation of these data will be done in a forthcoming paper [17]. A model which is capable to describe the drastic reduction of the integrated intensities beyond the solubility limit has to use information about the mobility of hydrogen in the metal matrix. Phenomenologically such an information is conveyed by the gas-liquid-solid model of some metal hydrides [18]. According to that model, phases exist which can be called interstitial solid

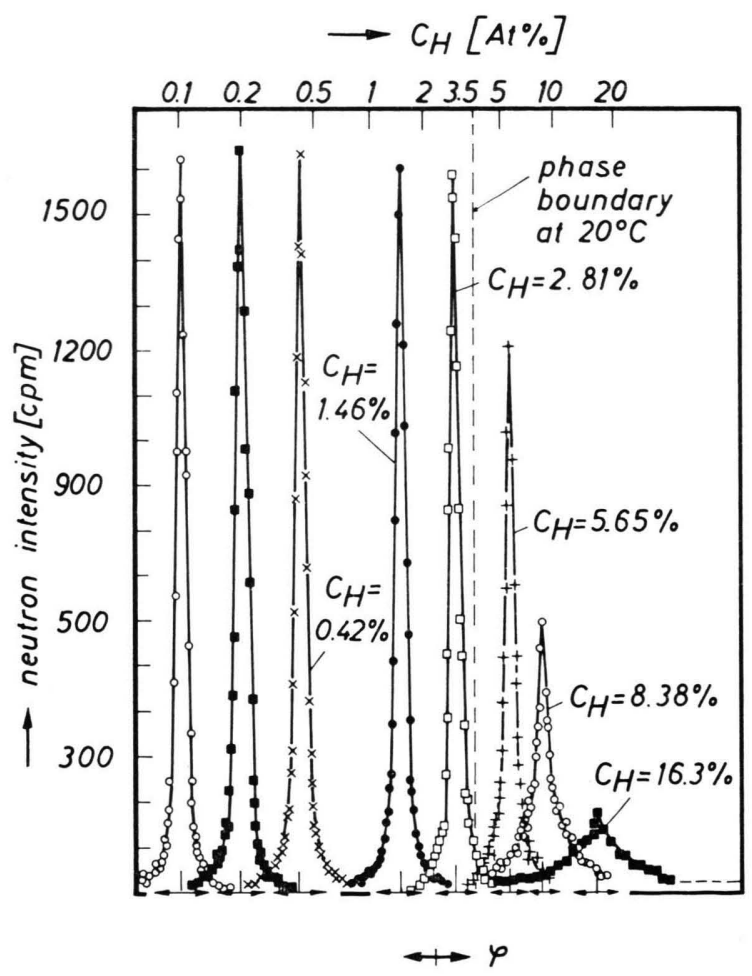

Fig. 3. Characteristic results for the hydrogen-niobium system. Observed rocking curves are plotted for various samples below and beyond the phase boundary at $20^{\circ} \mathrm{C}$. 


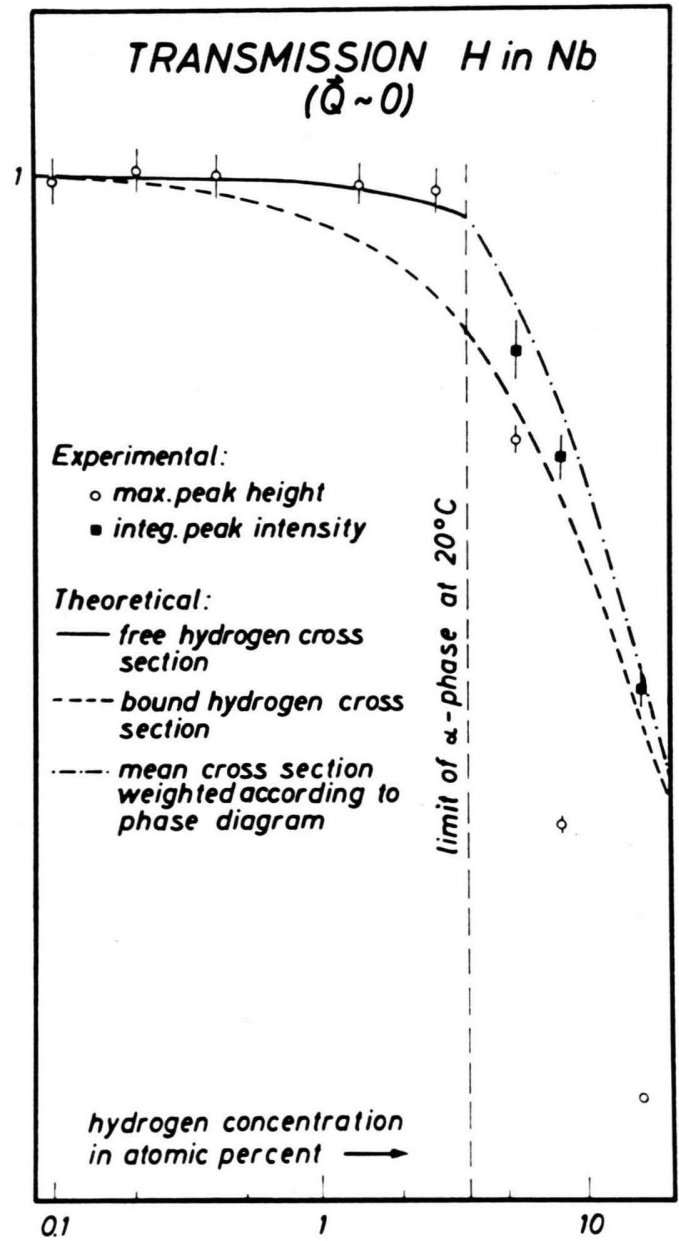

Fig. 4. Integial and peak intensity for hydrogen-niobium samples as a function of the hydrogen concentration.

solutions or interstitial alloys. Within the framework of that model the hydrogen in these metal-hydrogen systems can be described as existing in various forms of gaseous, liquid or conventional solid phases. In all systems relevant for the present work (Nb-H, V-H, V-D) the so-called $\alpha$-phase, which exists at room temperature at low $\mathrm{H}$ (or $\mathrm{D}$ ) concentrations up to a few atomic percent, exhibits a very high mobility of $\mathrm{H}$ (or D). It is this phase which corresponds to the "lattice gas". At higher - but not too high - concentrations precipitation of the $\beta$-phase occurs. In all cases considered here this phase is an ordered interstitial solid solution with correspondingly lower bulk mobility of the hydrogen isotopes.
In view o the phase diagram discussed above the experimental integrated peak intensity data below the solubility limit were compared with two different models (Fig. 4) corresponding to two extreme cases. One model assumes the hydrogen to be rigidly bound in the metal, while to other employs the free hydrogen crosssection equivalent to a monatomic gas. As is well known, these differ by a factor of 4 in the case of hydrogen and by a factor of 2.25 for the case of deuterium. As can easily be seen, the lattice gas model fits the experimental data quite well, while the assumption of a rigid bound disagrees with these data sigificantly. It is important to note that for $\mathrm{H}$ the total removal cross section is overwhelmingly caused by incoherent scattering.

On a more formal basis, we may employ the total cross section of a gas of noninteracting nuclei, i. e. a gas whose phase space density is sufficiently low for disregarding quantum interaction effects. For such a gas the total scattering cross section may be expressed as [19]

$$
\sigma=\overline{b^{2}} \frac{4 \pi}{\mu^{2}}\left\{\left(1+\frac{1}{2 \chi}\right) \varphi\left(\chi^{1 / 2}\right)+(\pi \chi)^{-1 / 2} e^{-\chi}\right\},
$$

with $\varphi(x)$ being the error integral

$$
p(x)=(2 \pi)^{-1 / 2} \int_{0}^{x} \mathrm{~d} t e^{-t^{2}} .
$$

Here, $b$ is the bound scattering length, $\mu=1+m / M$ and $\chi=E M \beta / m$, where $m$ is the neutron mass, $E$ is its kinetic energy, $M$ the mass of the scattering nuclei and $\beta=1 / k T$ describes the temperature of the

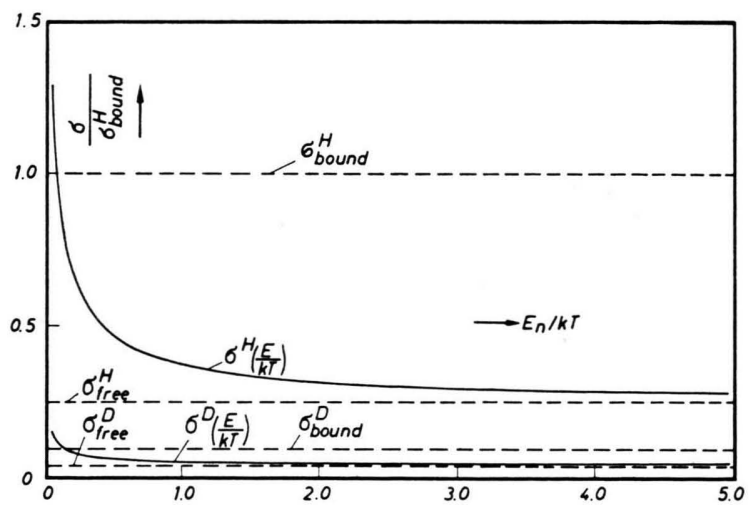

Fig. 5. Energy dependence of the hydrogen and deuterium attenuation cross section within the gas model. 
gas. The resulting cross section is shown in Fig. 5 for both hydrogen and deuterium. We note that for high neutron energies, i. e. $\chi \gg 1$, (3) reduces to the familiar

$$
\sigma \approx 4 \pi \overline{b^{2}}\left(\frac{A}{A+1}\right)^{2}
$$

where $A$ is the atomic mass of the scattering nuclei. Further support is given to our model by the property that beyond the solubility limit (Fig. 4) the integrated coherent small angle peak intensities are best explained by calculating a weighted mean between the bound and the free hydrogen cross sections. The weights there reflect the relative amounts of $\alpha$ and $\beta$ phase. These percentages were taken from the known phase diagrams [20].

Turning now to the V-H system, we observe a behavior very similar to the one in $\mathrm{Nb}-\mathrm{H}$. Here again the integrated intensity below the solubility limit is best explained by a lattice gas model (Figure 6). Above that limit the weighted mean approach gives again reasonable agreement with experiment. In the V-D system two important differences as to the cases discussed already are to be regarded. On the one hand the incoherent and therefore the total scattering cross section is significantly lower than that of $\mathrm{H}$, and on the other hand the $\left(\frac{A}{A+1}\right)^{2}$ factor is only 2.25 as mentioned above. Both these effects result in the fact that within the present experiment it was not possible to discriminate experimentally between the two cases.

[1] C. G. Shull, Phys. Rev. 179, 752 (1969).

[2] C. S. Schneider and C. G. Shull, Phys. Rev. B3, 830 (1971).

[3] S. Sh. Shilstein, V. A. Somenkov, and M. Kalanov, Sov. Phys. JETP 36, 1170 (1973) and Sov. Phys. Solid State 18, 1886 (1976).

[4] O. Schärpf and H. Strothmann, Proc. Conf. Neutr. Scatt. Gatlinburg, TN, CONF-760601-P2, Vol. II, p. 713 (1976).

[5] R. J. Weiss, Phys. Rev. 83, 379 (1951).

[6] M. Kalanov, S. Sh. Shilstein, and V. A. Somenkov, Sov. Phys. Cryst. 18, 755 (1974).

[7] D. Bader, Thesis, Technische Universität Wien 1981.

[8] W. H. Zachariasen, Theory of X-Ray Diffraction in Crystals, Dover Publ. Inc., New York 1967.

[9] H. Rauch and D. Petrascheck, Chapt. 9 in "Neutron Diffraction" (Ed. H. Dachs), Top. Curr. Phys. 6, 303 (1978).

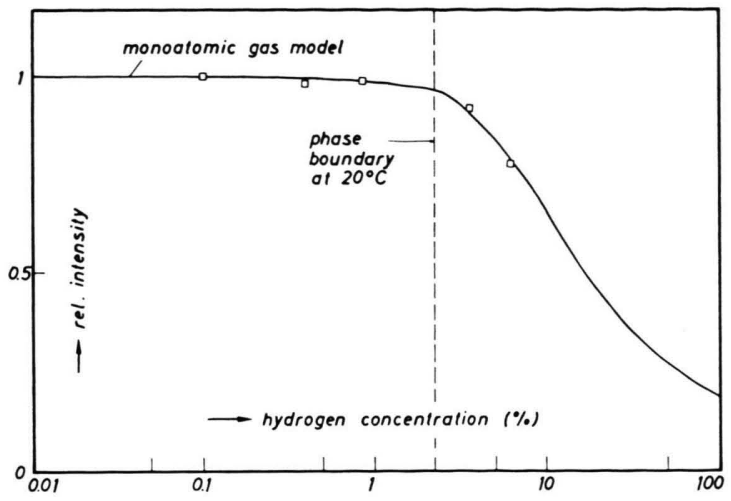

Fig. 6. Integral intensity for hydrogen-vanadium samples as a function of the hydrogen concentration.

\section{Concluding Comments}

The present experiments, though performed in the neutron optical regime, give some novel type of information about the mobility of $\mathrm{H}$ in metals. We point out that all this information may be obtained within a reasonable measurement time at a low flux reactor. Nevertheless, it would be of interest to complement the present work by classical small angle scattering studies as well as by a detailed exploitation of the information provided by the neutron small angle scattering in the optical regime.

\section{Acknowledgement}

The authors like to thank Dr. E. Seidl for many fruitful discussions and for his support during the sample preparation procedure and H. Bittermann for technical assistance.

[10] U. Bonse and M. Hart, Appl. Phys. Lett. 7, 238 (1965).

[11] U. Bonse and M. Hart, Z. Phys. 189, 151 (1966).

[12] Z. Szmid, B. Ler, A. Deluga, R. Bubákova, J. Drahokoupil, and A. Fingerland, Electron Techn. 6, 31 (1973).

[13] C. S. Schneider, Rev. Sci. Instrum. 44, 1594 (1973).

[14] H. Rauch, E. Seidl, A. Zeilinger, W. Bauspiess, and U. Bonse, J. Appl. Phys. 49, 2731 (1978).

[15] P. W. Schmidt and B. A. Fedorov, J. Appl. Cryst. 11, 411 (1978).

[16] O. Glatter, Acta Phys. Austr. 52, 243 (1980).

[17] W. Wachter, H. Rauch, and E. Seidl, in preparation.

[18] G. Alefeld, phys. stat. sol. 32, 67 (1969).

[19] W. Marshall and S. W. Lovesey, Theory of Thermal Neutron Scattering, Oxford Univ. Press, London 1971.

[20] T. Schober and H. Wenzel, Chapt. 2 in "Hydrogen in Metals II" (Ed. G. Alefeld and J. Völkl), Top. Appl. Phys. 29, 11 (1978), Springer-Verlag, Berlin 1978. 